\title{
Didelphys uterus with recurrent preterm labour: case report
}

\author{
Ibrahim Abdelazim ${ }^{1,2}$, Mohannad AbuFaza² \\ 'Department of Obstetrics and Gynecology, Ain Shams University, Cairo, Egypt \\ 2Department of Obstetrics and Gynecology, Ahmadi hospital, Kuwait Oil Company (KOC), Ahmadi, Kuwait
}

\begin{abstract}
Corresponding author: Ibrahim Abdelazim, Department of Obstetrics and Gynecology, Ain Shams University, Cairo, Egypt, phone: +965-66551300, e-mail: dr.ibrahimanwar@gmail.com
\end{abstract}

Müllerian anomalies (MAs) are congenital defects of the female genital tract due to failure of development of the Müllerian ducts, which normally occur in utero between 6 and 22 weeks [1].

The prevalence of MAs is about $4.3 \%$ in the general fertile population, $3.5 \%$ in the infertile population, and $13 \%$ in women with recurrent pregnancy losses (RPL) [2].

Septate uterus is the commonest type of MA (35\% incidence), followed by bicornuate uterus ( $25 \%$ incidence), and arcuate uterus (20\% incidence) [1].

Women with MAs are at risk of adverse pregnancy outcome, including recurrent pregnancy loss (RPL), low birth weight, preterm labour (PTL), malpresentation, and increased caesarean section rate $[3,4]$.

A 32-year-old woman, with one previous caesarean section (CS) at 35 weeks' gestation due to didelphys uterus (Figure 1) diagnosed during the first caesarean section, presented with recurrent PTL at 34 weeks +2 days, and breech presentation after uneventful antenatal care.

The PTL was diagnosed by regular uterine contractions $\geq 4$ contractions $/ 30 \mathrm{~min}$, each contraction lasting $>30-45 \mathrm{~s}$, with significant cervical changes on digital examination (cervical dilatation $>4 \mathrm{~cm}$ and $>50 \%$ effacement) [5].

During the antenatal care dexamethasone and magnesium sulphate $\left(\mathrm{MgSO}_{4}\right)$ were given to the studied woman at 28 weeks' gestation for foetal lung maturity and neuroprotection, respectively, because of risk of recurrent PTL (previous PTL and MA) [6-8].
The studied woman was given pre-operative prophylactic antibiotics according to the hospital protocol after exclusion of urinary tract infection, sexually transmitted diseases, and group B streptococcus infection [9]. She delivered by CS due to breech presentation a live baby boy, $2.230 \mathrm{~kg}$, with an Apgar score of 7, 8, and 9 at 1, 5, and $10 \mathrm{~min}$, respectively. The studied woman and her neonate were discharged from the hospital on the third postoperative day in good general condition.

Didelphys uterus occurs due to complete failure of fusion of the two Müllerian ducts, leading to two separate uterine cavities, two cervices, and two vaginas separated by a longitudinal vaginal septum [1].

Didelphys uterus is the least common type of MA, and it occurs in $8.3 \%$ of cases [1]. Didelphys uterus is suspected when the routine speculum examination showes two vaginal orifices separated by a longitudinal vaginal septum $[1,3]$.

A 2D ultrasound is usually used as the first imaging tool for diagnosis of MA followed by 3D ultrasound as a confirmatory tool $[1,3]$.

Magnetic resonance imaging (MRI) is an accurate tool in diagnosing MAs as hysterosalpingogram, hysteroscopy, and laparoscopy [1].

MAs are frequently associated with Wolffian duct anomalies (anomalies of the urinary tract) [1]. Vaz et al. concluded that MAs are associated with adverse fertility, and pregnancy outcomes in the form of RPL, low birth weight, PTL, malpresentation, and increased CS rate [3]. 


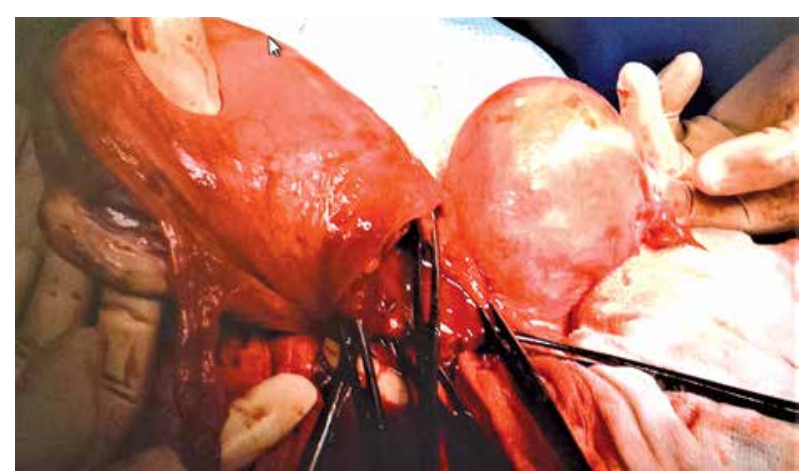

Figure 1. Intra-operative image during the caesarean section shows the didelphys uterus

In addition, Khander et al. concluded that women with MAs and prior PTL are at risk of recurrent PTL [10].

Similarly, the studied case had a history of PTL at 35 weeks and presented with recurrent PTL at 34 weeks +2 days, and delivered by CS for the second time due to breech presentation.

In conclusion, didelphys uterus is the least common type of Müllerian anomaly, and it occurs in $8.3 \%$ of cases. Women with didelphys uterus and Müllerian anomalies are at risk of preterm labour, malpresentation, and increased CS rate.

\section{Acknowledgments}

Authors are grateful to the studied woman who agreed and gave consent to use her dada for publication.

\section{Conflict of interest}

The authors declare no conflict of interest.

\section{References}

1. Rezai S, Bisram P, Lora Alcantara I, et al. Didelphys uterus: a case report and review of the literature. Case Rep Obstet Gynecol 2015; 2015: 865821.

2. Grimbizis GF, Camus M, Tarlatzis BC, et al. Clinical implications of uterine malformations and hysteroscopic treatment results. Hum Reprod Update 2001; 7: 161-74.

3. Vaz SA, Dotters-Katz SK, Kuller JA. Diagnosis and management of congenital uterine anomalies in pregnancy. Obstet Gynecol Surv 2017; 72: 194-201.

4. Lovelace D. Congenital uterine anomalies and uterine rupture. J Midwifery Womens Health 2016; 61: 501-6.

5. Abu-Faza M, Abdelazim IA, Svetlana S, et al. Diagnostic accuracy of premaquick versus actim partus in prediction of preterm labor in symptomatic women within 14 days. Open J Obstetr Gynecol 2018; 8: 741-55.

6. Al-Fadhli W, Al-Munaifi S, Abdelazim IA. Congenital diaphragmatic hernia: antenatal diagnosis and successful repair in preterm neonate case report. Gynecol Obstet Reprod Med 2018; 24: 104-7.

7. Abu-Faza M, Abdelazim IA. Antenatal magnesium sulphate (MgSO4) for fetal neuroprotection prior to preterm labor: mini-review. ARC J Gynecol Obstet 2017; 2: 11-4.
8. Farghali M, Abdelazim I, Abdelrazek K. Delayed second twin delivery: benefits and risks. J Matern Fetal Neonatal Med 2019; 32: 1626-32.

9. Abdulkareem A, Abdelazim IA. Pregnancy outcome after hysteroscopic synechiolysis for intrauterine synechiae (Asherman's syndrome): case report. J Obstet Gynecol Investig 2018; 1: 13-6.

10. Khander A, Stern E, Gerber RS, Fox NS. The association between obstetrical history and preterm birth in women with uterine anomalies. J Matern Fetal Neonatal Med 2018; 31: 2550-4. 